\title{
An Improved Large Area Silicon Drift Detector EDS System for Low Energy $X$-ray Detection and Fast Spectrum Imaging
}

\author{
L. Feng*, V. D. Saveliev*, M. Takahashi*, C. R. Tull*, S. Barkan*, E. V. Damron*, S. Kosuge*, \\ R. D. Lamb**, K. C. Whitherspoon** and Per O. Sjoman** \\ *SII NanoTechnology USA, Inc., 19865 Nordhoff Street, Northridge, CA 91324, USA \\ **IXRF Systems, Inc., 15715 Brookford Drive, Houston, TX 77059, USA
}

This paper reports on our recent efforts leading to significant improvements of our large area Vortex-EM ${ }^{\circledR}$ silicon drift detectors (SDD) [1] in low energy X-ray detection, energy resolution and peak-to-background ratio. These improvements, plus their already proven high throughput capabilities [2], make the Vortex-EM ${ }^{\circledR}$ SDD an ideal choice for today's electron microanalysis applications including elemental chemistry, fast spectrum imaging and high throughput particle analysis.

The new Vortex-EM ${ }^{\circledR}$ SDDs have a circular active area of $50 \mathrm{~mm}^{2}$ with an improved entrance window that enables detection of low energy x-rays down to Beryllium (Fig. 1a). In the detector package, an internal collimator is used to ensure best performance. Carbon peak distortions observed with earlier versions have been eliminated. The carbon peak resolution achieved is $\sim 67 \mathrm{eV}$ FWHM at $16 \mu$ s processor peaking time (Fig. 1b). At the same peaking time, the ${ }^{55} \mathrm{Fe}$ $\mathrm{Mn} \mathrm{K}_{\alpha} \mathrm{FWHM}$ is $\sim 128 \mathrm{eV}$ with a peak-to-background ratio of $\sim 18000$.

Combined with the EDS2008 microanalysis system of IXRF Systems, Inc., the Vortex-EM ${ }^{\circledR}$ SDD comes as the core of a unique EDS system - the e-Xpress ${ }^{\mathrm{TM}}$ which inherits all major state-of-theart features and functionality of the EDS2008 system in both hardware and software. One of the most important features of this system is its fast spectrum imaging capability, which adopts the latest event streaming technique that binds event information with position information in real time, allowing for acquisition of a complete spectrum pixel by pixel. The spectrum data of each pixel is saved with the maps and is conveniently retrievable from the map file. The hardware uses multiple buffers for seamless data transfer through high speed Ethernet communication, with an achievable data transfer rate of $\sim 2.5$ million events per second. The high output rate $(>300 \mathrm{kcps})$ at short peaking times of the Vortex-EM ${ }^{\circledR}$ SDD makes it possible to acquire maps at very high speed. Figure 2 shows $128 \times 128$ maps of a metal particle sample, acquired in 120 seconds. Also shown is the whole spectrum from a selected pixel in the map. Since the maps shown were acquired at an output rate much less than the maximum achievable rate, it would be possible to acquire them with a shorter dwell time and less frames under optimum conditions and thus the total acquire time could be well less than a minute. All the data presented here were collected on a TESCAN Vega II scanning electron microscope.

\section{References}

[1] S. Barkan, V. Saveliev, J. Iwanczyk, L. Feng, C. Tull, B. Patt, D. Newbury, J. Small, N. Zaluzec, "A New Improved Silicon Multi-Cathode Detector (SMCD) for Microanalysis and XRay Mapping Applications”, Microscopy Today, Vol. 12 (2004) 36-37. 
[2] L. Feng, J. Iwanczyk, B. Patt, S. Barkan and C. Tull, "A New High Performance Silicon Multi-cathode Detector for XRD and XRF Applications", Hard X-ray and Gamma-ray Detector Physics V, Proceedings of SPIE, International Society of Optical Engineering, Vol. 5198 (2004) 103-110.

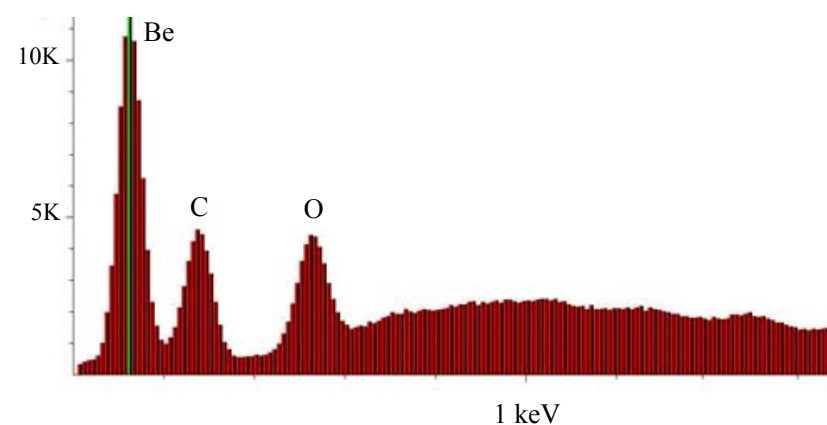

(a)

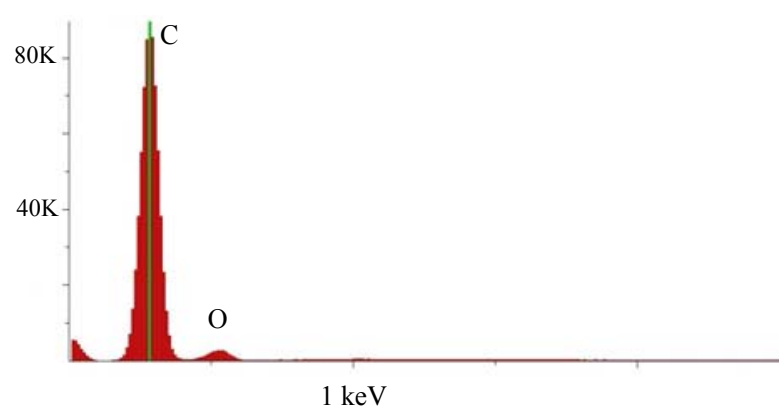

(b)

Fig. 1. Low energy spectra: (a) Be, $15 \mathrm{kV}, 800 \mathrm{pA}, 32 \mu$ s peaking time; (b) $\mathrm{C}, 15 \mathrm{kV}, 420 \mathrm{pA}$, $16 \mu$ s peaking time, peak $\mathrm{FWHM}=\sim 67 \mathrm{eV}$.

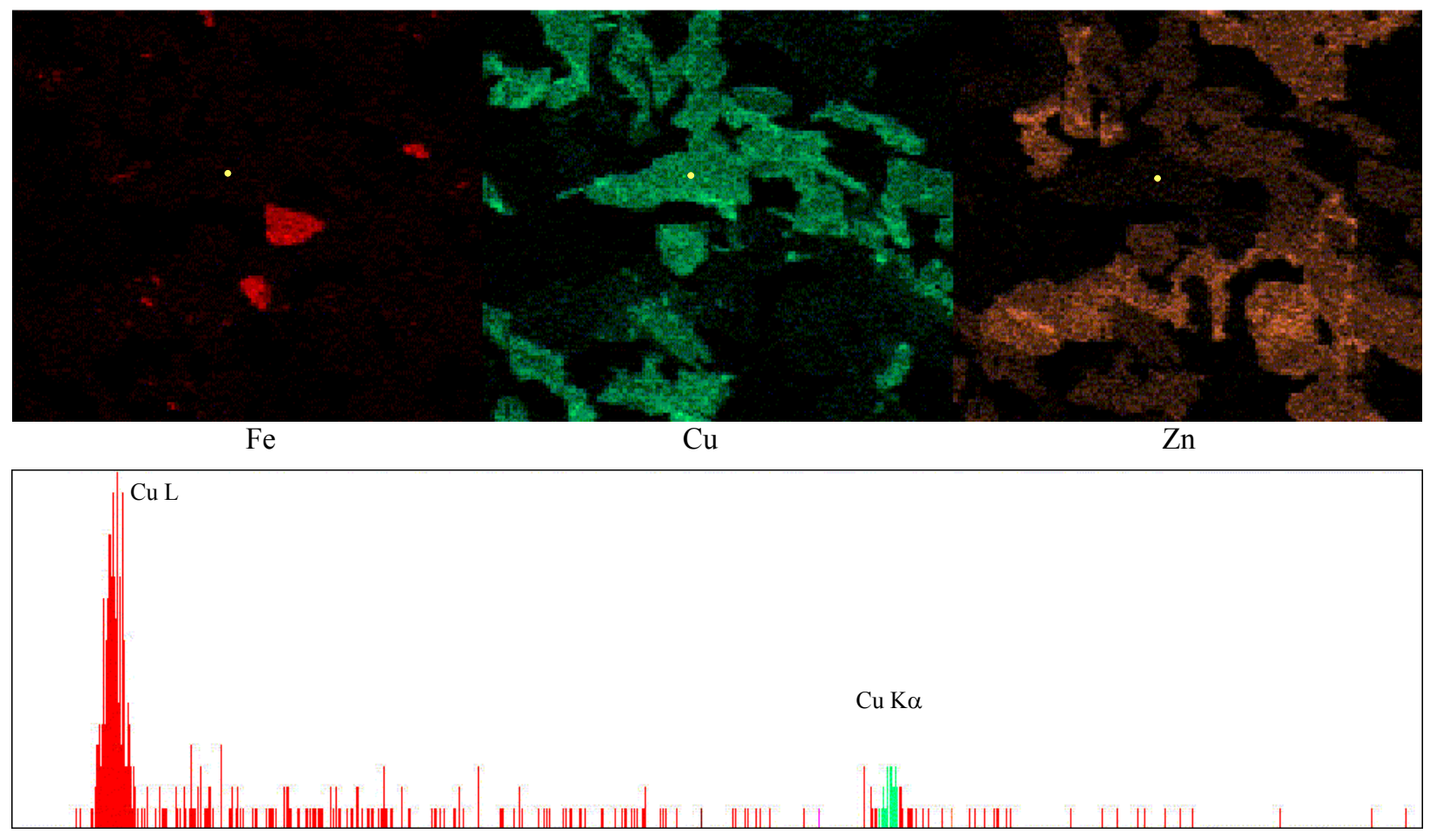

Fig. 2. Fast maps of metal particles on carbon tape: 300x magnification, 128x128 map size, $20 \mu \mathrm{s}$ dwell time, 100 frames; $15 \mathrm{kV}, 10 \mathrm{nA}, 1 \mu \mathrm{m}$ spot size; $0.5 \mu$ s processor peaking time, $70 \mathrm{kcps}$ output rate; total acquire time -120 seconds. The lower figure shows the spectrum retrieved for a selected pixel (the bright spots in the maps) from the map file. 